\title{
Nonlinear Dynamic in an Ecological System with Impulsive Effect and Optimal Foraging
}

\author{
Min Zhao ${ }^{1,2}$ and Chuanjun Dai ${ }^{1,2}$ \\ ${ }^{1}$ School of Life and Environmental Science, Wenzhou University, Wenzhou, Zhejiang 325035, China \\ ${ }^{2}$ Zhejiang Provincial Key Laboratory for Water Environment and Marine Biological Resources Protection, Wenzhou University, \\ Wenzhou, Zhejiang 325035, China
}

Correspondence should be addressed to Min Zhao; zmcn@tom.com

Received 13 January 2014; Accepted 15 May 2014; Published 16 June 2014

Academic Editor: Weiming Wang

Copyright (C) 2014 M. Zhao and C. Dai. This is an open access article distributed under the Creative Commons Attribution License, which permits unrestricted use, distribution, and reproduction in any medium, provided the original work is properly cited.

\begin{abstract}
The population dynamics of a three-species ecological system with impulsive effect are investigated. Using the theories of impulsive equations and small-amplitude perturbation scales, the conditions for the system to be permanent when the number of predators released is less than some critical value can be obtained. Furthermore, because the predator in the system follows the predictions of optimal foraging theory, it follows that optimal foraging promotes species coexistence. In particular, the less beneficial prey can support the predator alone when the more beneficial prey goes extinct. Moreover, the influences of the impulsive effect and optimal foraging on inherent oscillations are studied using simulation, which reveals rich dynamic behaviors such as period-halving bifurcations, a chaotic band, a periodic window, and chaotic crises. In addition, the largest Lyapunov exponent and the power spectra of the strange attractor, which can help analyze the chaotic dynamic behavior of the model, are investigated. This information will be useful for studying the dynamic complexity of ecosystems.
\end{abstract}

\section{Introduction}

In recent years, interest in studying nonlinear dynamic systems has exploded. In the 1970s, since the pioneering work of May on the relationship between food-web complexity and stability and the chaotic phenomenon [1-3], more and more researchers have become interested in dynamic behavior involving ecological mechanisms that promote species diversity [4-20]. More recently, dynamic systems' studies have benefited from an infusion of interest and new techniques in ecology.

It is known that when a predator is shared by two noncompeting species, predator-mediated apparent competition often leads to competitive exclusion of one prey population [21]. This phenomenon is related to optimal foraging and adaptive foraging. A two-prey-one-predator population model with optimal predator foraging behavior has been studied in a fine-grained environment [22-24]. On this basis, Krrivan and Eisner considered a system composed of two prey types and an optimally foraging predator [25] in a system described by the following model:

$$
\begin{array}{r}
\dot{x}(t)=x(t)\left(r_{1}(x(t))\right. \\
\left.\quad-\frac{\lambda_{1} z(t)}{1+h_{1} \lambda_{1} x(t)+u h_{2} \lambda_{2} y(t)}\right) \\
\dot{y}(t)=y(t)\left(r_{2}(y(t))\right. \\
\left.\quad-\frac{u \lambda_{2} z(t)}{1+h_{1} \lambda_{1} x(t)+u h_{2} \lambda_{2} y(t)}\right) \\
\dot{z}(t)=z(t)\left(\frac{e_{1} \lambda_{1} x(t)+u e_{2} \lambda_{2} y(t)}{1+h_{1} \lambda_{1} x(t)+u h_{2} \lambda_{2} y(t)}-m\right) .
\end{array}
$$

This paper considers an impulsive differential-equation model based on model (1), which assumes that predators 
forage according to optimal foraging theory [23, 24]. This system can be expressed by the following equations:

$$
\begin{gathered}
\dot{x}(t)=r_{1} x(t)\left(\frac{k_{0}-x(t)}{k_{1}-x(t)}\right)-b_{1} x^{2}(t) \\
-\frac{\lambda_{1} x(t) z(t)}{1+h_{1} \lambda_{1} x(t)+u h_{2} \lambda_{2} y(t)} \\
\dot{y}(t)=r_{2} y(t)\left(\frac{k_{2}-y(t)}{k_{3}-y(t)}\right)-b_{2} y^{2}(t) \\
-\frac{u \lambda_{2} y(t) z(t)}{1+h_{1} \lambda_{1} x(t)+u h_{2} \lambda_{2} y(t)} \\
\left.\dot{z}(t)=z(t)\left(\begin{array}{c}
e_{1} \lambda_{1} x(t)+u e_{2} \lambda_{2} y(t) \\
1+h_{1} \lambda_{1} x(t)+u h_{2} \lambda_{2} y(t)
\end{array}\right) m\right) \\
t \neq n T \\
x\left(t^{+}\right)=x(t) \\
y\left(t^{+}\right)=y(t) \\
z\left(t^{+}\right)=z(t)+p \\
t=n T,
\end{gathered}
$$

where $x(t), y(t), z(t)$ are, respectively, the densities of two prey types and one predator at time $t, r_{i}(i=1,2)$ is the per capita prey intrinsic growth rate, $r_{1} \cdot k_{0}\left(0 \leq k_{0} / k_{1} \leq\right.$ $1), r_{2} \cdot k_{2}\left(0 \leq k_{2} / k_{3} \leq 1\right)$ are the respective carrying capacities of the prey, and $k_{1}, k_{3}$ are the corresponding values of available resources or in the ideal case (i.e., where no resources are wasted) the carrying capacity. However, the ideal case is impossible in reality. The ratios $k_{0} / k_{1}, k_{2} / k_{3}$ express the relative efficiency of nutrient utilization in species $x, y$. At any time, if $x<k_{0}<k_{1}, y<k_{2}<k_{3}$, the efficiency is high as long as $k_{0} / k_{1}, k_{2} / k_{3}$ are close to one; when the values are lower, this indicates that resource limitations are restricting the population increase [23]. $b_{i}(i=1,2)$ are the rate of intraspecific competition of the prey, $\lambda_{i}(i=1,2)$ is the cropping rate of a predator feeding on the $i$ th prey type, $e_{i}(i=1,2)$ is the conversion factor relating predator reproduction to prey consumption, and $h_{i}(i=1,2)$ is the per capita mortality rate for the forager. In this paper, it is assumed that prey type $x$ is more beneficial than the other and hence $e_{1} / h_{1}>e_{2} / h_{2}[26,27]$. To study optimal foraging, a control parameter $u(0 \leq u \leq 1)$ is introduced [25], which represents the probability that the alternative second prey type is included in the predator's diet. $T$ is the period of the impulsive effect, $n \in N$, and $p>0$ is the number of predators released at $t=n T$. To achieve a set of conditions which can guarantee that the system will be permanent and that the numbers of the two prey types are not so large that they go extinct because of exceeding the carrying capacity of the environment, the model will release a certain number of predators only at $t=n T$ because the predator is assumed to be a versatile and advanced predator.

The rest of this paper is organized as follows. Section 2 will review the effect of impulsive perturbations, establish conditions for extinction, and obtain the conditions for permanence of System (2) using the Floquet theory of impulsive equations at small-amplitude perturbation scales. In Section 3, the results of computer-based numerical analysis are shown and discussed briefly. In addition, the largest Lyapunov exponent, which also indicates the chaotic dynamic behavior of the model, is computed, and the Fourier spectra, which illustrate the qualitative nature of strange attractors, are plotted. Finally, conclusions and remarks are stated.

\section{Analysis of the System}

Let $R_{+}=[0, \infty), R_{+}=\left\{X \in R^{3}: X \geq 0, X=(x, y, z)\right\}$, $\Omega=\operatorname{int} R_{+}^{3}$, and let $N$ be the set of all nonnegative integers. The map $g=\left(g_{1}, g_{2}, g_{3}\right)^{T}$ is defined by the right-hand side of the first three equations of System (2).

Let $V: R_{+} \times R_{+}^{3} \rightarrow R_{+}$; then $V$ is said to belong to class $V_{0}$ if

(1) $V$ is continuous in $(n T,(n+1) T] \times R_{+}^{3}$, and for each $x \in R_{+}^{3}, n \in N, \lim _{(t, y) \rightarrow\left(n T^{+}, x\right)} V(t, y)=V\left(n T^{+}, x\right)$ exists;

(2) $V$ is locally Lipschitzian in $X$.

Definition 1. Let $V \in V_{0}$; then, for $(t, x) \in(n T,(n+1) T] \times$ $R_{+}^{3}$, the upper right derivative of $V(t, x)$ with respect to the impulsive differential System (2) can be defined as

$$
D^{+} V(t, x)=\lim _{h \rightarrow 0} \sup \frac{1}{h}[V(t+h, x+h g(t, x))-V(t, x)] \text {. }
$$

The solution of System (2) is a piecewise continuous function $X: R_{+} \rightarrow R_{+}^{3}$, where $X(t)$ is continuous on $(n T,(n+$ 1)T], $n \in N$, and $X\left(n T^{+}\right)=\lim _{t \rightarrow n T} X(t)$ exists. Obviously, the smoothness property of $g$ guarantees the global existence and uniqueness of a solution of System (2) (for details, see [28-30]).

Definition 2. System (2) is said to be permanent if there exists a compact region $\Omega=$ int $R_{+}^{3}$ such that every solution $(x(t), y(t), z(t))$ of System (2) will eventually enter and remain in the region $\Omega$.

The following lemma will now be presented.

Lemma 3. Let $X(t)$ be a solution of System (2) with $X\left(0^{+}\right) \geq 0$; then $X(t) \geq 0$ for all $t \geq 0$, and furthermore $X(t)>0, t \geq 0$ if $X\left(0^{+}\right)>0$.

An important comparison theorem will now be used on the impulsive differential equation.

Lemma 4 (see [28-30]). Let $V \in V_{0}$ and assume that

$$
\begin{array}{cc}
D^{+} V(t, X) \leq f(t, V(t, X)) & t \neq n T \\
V\left(t, X\left(t^{+}\right)\right) \leq \varphi_{n}(V(t, X)) & t=n T,
\end{array}
$$

where $f: R_{+} \times R_{+} \rightarrow R$ is continuous in $(n T,(n+1) T] \times R_{+}$and for $\mu \in R_{+}, n \in N, \lim _{(t, y) \rightarrow\left(n T^{+}, \mu\right)} f(t, y)=f\left(n T^{+}, \mu\right)$ exists 
and $\varphi_{n}: R_{+} \rightarrow R_{+}$is nondecreasing. Let $r(t)$ be the maximal solution to the scalar impulsive differential equation

$$
\begin{gathered}
\dot{u}(t)=f(t, u(t)) \quad t \neq n T \\
u\left(t^{+}\right)=\varphi_{n}(u(t)) \quad t=n T \\
u\left(0^{+}\right)=u_{0}
\end{gathered}
$$

existing on $[0, \infty)$. Then $V\left(0^{+}, X_{0}\right) \leq u_{0}$ implies that $V(t, X(t)) \leq r(t), t \geq 0$, where $X(t)$ is any solution to System (2).

Theorem 5. There exists a constant $M$ such that $x(t) \leq$ $M, y(t) \leq M$, and $z(t) \leq M$ for each solution $X(t)=$ $(x(t), y(t), z(t))$ of System (2) for all t large enough.

Proof. Define $V(t, X(t))$ such that

$$
V(t, X(t))=e_{1} x(t)+e_{2} y(t)+z(t)
$$

where $V \in V_{0}$. Since $\dot{x}(t) \leq r_{1} x(t)-b_{1} x^{2}(t)$ and $\dot{y}(t) \leq$ $r_{2} y(t)-b_{2} y^{2}(t)$, then $x(t) \leq r_{1} / b_{1}, y(t) \leq r_{2} / b_{2}$, and the upper right derivative of $V(t, X(t))$ can be calculated along a solution of System (2), yielding the following impulsive differential equation:

$$
\begin{aligned}
D^{+} V(t)+L V(t)= & L e_{1} x(t)+r_{1} e_{1} x(t)\left(\frac{k_{0}-x(t)}{k_{1}-x(t)}\right) \\
& -b_{1} e_{1} x^{2}(t)+L e_{2} y(t) \\
& +r_{2} e_{2} y(t)\left(\frac{k_{2}-y(t)}{k_{3}-y(t)}\right)-b_{2} e_{2} y^{2}(t) \\
& +(L-m) z(t) \quad t \neq n T \\
V\left(t^{+}\right) & =V(t)+p \quad t=n T .
\end{aligned}
$$

Obviously,

$$
\begin{aligned}
D^{+} V(t)+L V(t) \leq & \left(L e_{1}+r_{1} e_{1}\right) x(t)-b_{1} e_{1} x^{2}(t) \\
& +\left(L e_{2}+r_{2} e_{2}\right) y(t)-b_{2} e_{2} y^{2}(t) \\
& +(L-m) z(t) .
\end{aligned}
$$

Let $0<L<m$; then $D^{+} V(t)+L V(t)$ is bounded. Select $L_{1}, L_{2}$ such that

$$
\begin{gathered}
D^{+} V(t) \leq-L_{1} V(t)+L_{2} \quad t \neq n T \\
V\left(t^{+}\right)=V(t)+p \quad t=n T,
\end{gathered}
$$

where $L_{1}, L_{2}$ are two positive constants.
According to Lemma 4,

$$
\begin{aligned}
V(t) \leq & \left(V\left(0^{+}\right)-\frac{L_{2}}{L_{1}}\right) \exp \left(-L_{1} t\right) \\
& +\frac{p \exp \left(1-\exp \left(-n L_{1} t\right)\right)}{\exp \left(L_{1} T\right)-1} \exp \left(L_{1} t\right) \\
& \times \exp \left(-L_{1}(t-n t)\right)+\frac{L_{2}}{L_{1}},
\end{aligned}
$$

where $t \in(n T,(n+1) T]$. Hence,

$$
\lim _{t \rightarrow \infty} V(t) \leq \frac{L_{2}}{L_{1}}+\frac{p \exp \left(L_{1} T\right)}{\exp \left(L_{1} T\right)-1} .
$$

Therefore, $V(t, X(t))$ is ultimately bounded, and it follows that each positive solution of System (2) is uniformly ultimately bounded. This completes the proof.

Next, some basic properties of the following subsystem of System (2), in which the two prey types are absent, will be defined:

$$
\begin{gathered}
\dot{z}(t)=-m z(t) \quad t \neq n T \\
z\left(t^{+}\right)=z(t)+p \quad t=n T \\
z\left(0^{+}\right)=z_{0} .
\end{gathered}
$$

Clearly, $z^{*}(t)=p \exp (-m(t-n T)) /(1-\exp (-m T)), t \in$ $(n T,(n+1) T], n \in N, z^{*}\left(0^{+}\right)=p /(1-\exp (-m T))$ is a positive periodic solution of System (12). Hence,

$$
z(t)=\left(z\left(0^{+}\right)-\frac{p}{1-\exp (-m T)}\right) \exp (-m T)+z^{*}(t)
$$

is a solution of System (12) with initial value $z_{0} \geq 0$, where $t \in(n T,(n+1) T], n \in N$.

Lemma 6. For a positive periodic solution $z^{*}(t)$ of System (12) and every solution $z(t)$ of System (12) with $z_{0} \geq 0, \mid z(t)-$ $z^{*}(t) \mid \rightarrow 0, t \rightarrow \infty$.

Hence, when only the predator is present, it is possible to obtain the complete expression for the periodic solution $\left(0,0, z^{*}(t)\right)$ of System (2).

Based on these discussions, the following theorems can be proved.

Theorem 7. Let $(x(t), y(t), z(t))$ be any solution of System (2). Then

(1) $\left(0,0, z^{*}(t)\right)$ is said to be locally asymptotically stable if $p>\max \left(r_{1} k_{0} T m / \lambda_{1} k_{1}, r_{2} k_{2} T m / u \lambda_{2} k_{3}\right)$;

(2) $\left(0,0, z^{*}(t)\right)$ is said to be globally asymptotically stable if $p>\max \left(r_{1} k_{0} T m / \lambda_{1} k_{1}, r_{2} k_{2} T m / u \lambda_{2} k_{3}\right)$ and

$$
\begin{aligned}
& \frac{p \exp (-m T)}{1-\exp (-m T)}>\max ( \frac{r_{1}\left(1+h_{1} \lambda_{1} M+u h_{2} \lambda_{2} M\right)}{\lambda_{1}}, \\
&\left.\frac{r_{2}\left(1+h_{1} \lambda_{1} M+u h_{2} \lambda_{2} M\right)}{u \lambda_{2}}\right) .
\end{aligned}
$$


Proof. The local stability of the periodic solution $\left(0,0, z^{*}(t)\right)$ may be determined by considering the behavior of smallamplitude perturbations of the solution. Define

$$
x(t)=u(t), \quad y(t)=v(t), \quad z(t)=w(t)+z^{*}(t) .
$$

Then substitute (15) into System (2). The linearization of the system becomes

$$
\begin{gathered}
\dot{u}(t)=\left(r_{1} \frac{k_{0}}{k_{1}}-\lambda_{1} z^{*}\right) u(t) \\
\dot{v}(t)=\left(r_{2} \frac{k_{2}}{k_{3}}-u \lambda_{2} z^{*}\right) v(t) \\
\dot{w}(t)=z^{*} e_{1} \lambda_{1} u(t)+z^{*} u e_{2} \lambda_{2} v(t)-m w(t) \\
u\left(t^{+}\right)=u(t) \\
v\left(t^{+}\right)=v(t) \\
w\left(t^{+}\right)=w(t) \\
t=n T .
\end{gathered}
$$

Therefore,

$$
\left(\begin{array}{c}
u(t) \\
v(t) \\
w(t)
\end{array}\right)=\Phi(t)\left(\begin{array}{c}
u(0) \\
v(0) \\
w(0)
\end{array}\right) \quad 0 \leq t \leq T
$$

where $\Phi(t)$ satisfies

$$
\frac{d \Phi}{d t}=\left(\begin{array}{ccc}
r_{1} \frac{k_{0}}{k_{1}}-\lambda_{1} z^{*} & 0 & 0 \\
0 & r_{2} \frac{k_{2}}{k_{3}}-u \lambda_{2} z^{*} & 0 \\
z^{*} e_{1} \lambda_{1} & z^{*} u e_{2} \lambda_{2} & -m
\end{array}\right) \Phi(t)
$$

and $\Phi(0)=I$, the identity matrix, and

$$
\left(\begin{array}{c}
u\left(n T^{+}\right) \\
v\left(n T^{+}\right) \\
w\left(n T^{+}\right)
\end{array}\right)=\left(\begin{array}{lll}
1 & 0 & 0 \\
0 & 1 & 0 \\
0 & 0 & 1
\end{array}\right)\left(\begin{array}{c}
u(n T) \\
v(n T) \\
w(n T)
\end{array}\right) .
$$

The stability of the periodic solution $\left(0,0, z^{*}(t)\right)$ is determined by the eigenvalues of

$$
\Theta=\left(\begin{array}{lll}
1 & 0 & 0 \\
0 & 1 & 0 \\
0 & 0 & 1
\end{array}\right) \Phi(T)
$$

which are

$$
\begin{aligned}
& \mu_{1}=\exp \left(\int_{0}^{T} r_{1} \frac{k_{0}}{k_{1}}-\lambda_{1} z^{*} d t\right), \\
& \mu_{2}=\exp \left(\int_{0}^{T} r_{2} \frac{k_{2}}{k_{3}}-u \lambda_{2} z^{*} d t\right) \\
& \mu_{3}=\exp (-m T)<1
\end{aligned}
$$

According to Floquet theory, $\left(0,0, z^{*}(t)\right)$ is locally asymptotically stable if $\left|\mu_{1}\right|<1$ and $\left|\mu_{2}\right|<1$; that is, $p>$ $\max \left(r_{1} k_{0} T m / \lambda_{1} k_{1}, r_{2} k_{2} T m / u \lambda_{2} k_{3}\right)$.

If $\left(0,0, z^{*}(t)\right)$ is locally asymptotically stable and a global attractor, then $\left(0,0, z^{*}(t)\right)$ is globally asymptotically stable. In the following, global attractiveness will be demonstrated.

Let $V(t)=x(t)+y(t)$; then

$$
\begin{aligned}
\left.\dot{V}(t)\right|_{(2)}= & r_{1} x(t)\left(\frac{k_{0}-x(t)}{k_{1}-x(t)}\right)-b_{1} x^{2}(t) \\
& -\frac{\lambda_{1} x(t) z(t)}{1+h_{1} \lambda_{1} x(t)+u h_{2} \lambda_{2} y(t)} \\
& +r_{2} y(t)\left(\frac{k_{2}-y(t)}{k_{3}-y(t)}\right)-b_{2} y^{2}(t) \\
& -\frac{u \lambda_{2} y(t) z(t)}{1+h_{1} \lambda_{1} x(t)+u h_{2} \lambda_{2} y(t)} \\
\left.\dot{V}(t)\right|_{(2)} \leq & \left(r_{1}-\frac{\lambda_{1} z(t)}{1+h_{1} \lambda_{1} x(t)+u h_{2} \lambda_{2} y(t)}\right) x(t) \\
& -b_{1} x^{2}(t) \\
& +\left(r_{2}-\frac{u \lambda_{2} z(t)}{1+h_{1} \lambda_{1} x(t)+u h_{2} \lambda_{2} y(t)}\right) y(t) \\
& -b_{2} y^{2}(t) .
\end{aligned}
$$

By Theorem 5, there exists a constant $M>0$ such that $x(t) \leq M, y(t) \leq M$, and $z(t) \leq M$ for each solution $X(t)=(x(t), y(t), z(t))$ of System (2) with all $t$ large enough. Therefore,

$$
\begin{gathered}
\dot{z}(t)=z(t)\left(\frac{e_{1} \lambda_{1} x(t)+u e_{2} \lambda_{2} y(t)}{1+h_{1} \lambda_{1} x(t)+u h_{2} \lambda_{2} y(t)}-m\right) \\
\geq-m z(t) \quad t \neq n T . \\
z\left(t^{+}\right)=z(t)+p \quad t=n T
\end{gathered}
$$

By Lemmas 4 and 6 , it is known that there exists $t_{1}>0$ and it is possible to select $\varepsilon>0$ small enough so that $z(t) \geq$ $z^{*}(t)-\varepsilon$. Therefore, for all $t \geq t_{1}$,

$$
\begin{aligned}
z(t) & \geq z^{*}(t)-\varepsilon \\
& =\frac{p \exp (-m(t-n T))}{1-\exp (-m T)}-\varepsilon \\
& \geq \frac{p \exp (-m T)}{1-\exp (-m T)}-\varepsilon
\end{aligned}
$$

Define

$$
\gamma=\frac{p \exp (-m T)}{1-\exp (-m T)}-\varepsilon
$$


Then $r_{1}-\left(\lambda_{1} \gamma /\left(1+h_{1} \lambda_{1} M+u h_{2} \lambda_{2} M\right)\right)<0$ and $r_{1}-$ $\left(u \lambda_{2} \gamma /\left(1+h_{1} \lambda_{1} M+u h_{2} \lambda_{2} M\right)\right)<0$. Thus, for $t \geq t_{1}$,

$$
\begin{aligned}
\left.\dot{V}(t)\right|_{(2)} \leq & \left(r_{1}-\frac{\lambda_{1} \gamma}{1+h_{1} \lambda_{1} M+u h_{2} \lambda_{2} M}\right) x(t)-b_{1} x^{2}(t) \\
& +\left(r_{2}-\frac{u \lambda_{2} \gamma}{1+h_{1} \lambda_{1} M+u h_{2} \lambda_{2} M}\right) y(t) \\
& -b_{2} y^{2}(t)<0 .
\end{aligned}
$$

So $V(t) \rightarrow 0, x(t) \rightarrow 0, y(t) \rightarrow 0$ as $t \rightarrow \infty$. Note that the limiting case of System (2) is exactly System (12) together with Lemma 6 . It follows that the periodic solution $\left(0,0, z^{*}(t)\right)$ is a global attractor. This completes the proof.

Theorem 8. System (2) is permanent if

$$
\begin{gathered}
p<\min \left(\frac{r_{1} k_{0} T m}{\lambda_{1} k_{1}}, \frac{r_{2} k_{2} T m}{u \lambda_{2} k_{3}}\right), \\
\frac{p \exp (-m T)}{1-\exp (-m T)}<\min \left(\frac{r_{1}\left(1+h_{1} \lambda_{1} M+u h_{2} \lambda_{2} M\right)}{\lambda_{1}},\right. \\
\left.\frac{r_{2}\left(1+h_{1} \lambda_{1} M+u h_{2} \lambda_{2} M\right)}{u \lambda_{2}}\right) .
\end{gathered}
$$

Proof. Let $X(t)=(x(t), y(t), z(t))$ be any solution of System (2) with $X(0)>0$. From Theorem 5, assume that $x(t) \leq$ $M, y(t) \leq M, z(t) \leq M$.

From System $(2), \dot{x}(t) \leq\left(r_{1}-b_{1} x(t)\right) x(t)$.

Consider the following equation:

$$
\begin{aligned}
& \dot{w}(t)=w(t)\left(r_{1}-b_{1} w(t)\right) \\
& w(0)=x(0) .
\end{aligned}
$$

It is possible to obtain $x(t) \leq w(t)$ and $w(t) \rightarrow r_{1} / b_{1}$ as $t \rightarrow \infty$. Hence, for any $\varepsilon_{1}>0, x(t)<r_{1} / b_{1}+\varepsilon_{1}$ for all $t$ sufficiently large. For simplification, it may be assumed that $x(t)<r_{1} / b_{1}+\varepsilon_{1}$ holds for all $t>0$. The same arguments can be made for any $\varepsilon_{2}>0, y(t)<r_{2} / b_{2}+\varepsilon_{2}$ for all $t>0$. Let $m_{3}=(p \exp (-m T)) /(1-\exp (-m T))-\varepsilon(\varepsilon>0)$. Note that $\dot{z}(t) \geq-m z(t)$, and consider the following equation:

$$
\begin{aligned}
\dot{w}(t) & =-b_{3} w(t) \quad t \neq n T \\
w\left(t^{+}\right) & =w(t)+p \quad t=n T \\
w\left(0^{+}\right) & =z\left(0^{+}\right)>0 .
\end{aligned}
$$

From Lemmas 4 and $6, z(t) \geq w(t)>z^{*}(t)-\varepsilon>0$, and hence $z(t)>m_{3}$ for all $t$ sufficiently large. Therefore, it is necessary to find $l_{1}>0$ and $l_{2}>0$ such that $x(t) \geq l_{1}$, $y(t) \geq l_{2}$ for all $t$ large enough. This can be done as shown in the following two steps.

First, choose $0<l_{1}<m / 2 e_{1} \lambda_{1}, 0<l_{2}<m / 2 u e_{2} \lambda_{2}$ such that $e_{1} \lambda_{1} l_{1}+u e_{2} \lambda_{2} l_{2}<m$. Then there exist $t_{1} \in(0, \infty)$ and $t_{2} \in(0, \infty)$ such that $x\left(t_{1}\right) \geq l_{1}, y\left(t_{2}\right) \geq l_{2}$. Otherwise,
(1) there exists a $t_{2}>0$ such that $y\left(t_{2}\right) \geq l_{2}$, but $x\left(t_{1}\right)<l_{1}$ for all $t>0$;

(2) there exists a $t_{1}>0$ such that $x\left(t_{1}\right) \geq l_{1}$, but $y\left(t_{2}\right)<l_{2}$ for all $t>0$;

(3) there are $x\left(t_{1}\right)<l_{1}$ and $y\left(t_{2}\right)<l_{2}$ for all $t>0$.

For case (1), according to Theorem 8 , select $\eta_{1}>0$ small enough so that

$$
\omega_{1}=\int_{n T}^{(n+1) T}\left(r_{1}-b_{1} l_{1}-\lambda_{1}\left(v^{*}+\eta_{1}\right)\right) d t>0 .
$$

From case (1),

$$
\begin{gathered}
\dot{z}(t) \leq\left(e_{1} \lambda_{1} x(t)+u e_{2} \lambda_{2} y(t)-m\right) z(t) \\
\leq\left(-m+e_{1} \lambda_{1} l_{1}+u e_{2} \lambda_{2}\left(\frac{r_{2}}{b_{2}}+\varepsilon_{1}\right)\right) z(t) \\
=A z(t) \quad t \neq n T \\
z\left(t^{+}\right)=z(t)+p \quad t=n T,
\end{gathered}
$$

where $A=-m+e_{1} \lambda_{1} l_{1}+u e_{2} \lambda_{2}\left(r_{2} / b_{2}+\varepsilon_{1}\right)$. Therefore, $z(t) \leq$ $v(t)$ and $v(t) \rightarrow v^{*}(t)$ as $t \rightarrow \infty$, where $v(t)$ is the solution of the following equation:

$$
\begin{gathered}
\dot{v}(t)=A v(t) \quad t \neq n T \\
v\left(t^{+}\right)=v(t)+p \quad t=n T \\
v\left(0^{+}\right)=z\left(0^{+}\right) \geq 0
\end{gathered}
$$

and $v^{*}(t)=p \exp (A(t-n T)) /(1-\exp (A T))$. Therefore, there exists a $T_{1}>0$, when $t>T_{1}$,

$$
\begin{gathered}
z(t) \leq v(t)<v^{*}(t)+\eta_{1}, \\
\dot{x}(t) \geq\left(r_{1}-b_{1} l_{1}-\lambda_{1}\left(v^{*}(t)+\eta_{1}\right)\right) x(t) \quad t \neq n T \\
x\left(t^{+}\right)=x(t) \quad t=n T .
\end{gathered}
$$

Let $N_{1} \in N$ and $N_{1} T \geq T_{1}$. Integrating (34) on ( $n T$, $(n+$ 1)T], $n \geq N_{1}$, the following result can be obtained:

$$
\begin{aligned}
& x((n+1) T) \\
& \quad \geq x(n T) \exp \left(\int_{n T}^{(n+1) T}\left(r_{1}-b_{1} l_{1}-\lambda_{1}\left(v^{*}+\eta_{1}\right)\right) d t\right) \\
& \quad=x(n T) \exp \left(\omega_{1}\right) .
\end{aligned}
$$

Then $x\left(\left(N_{1}+k\right) T\right) \geq x\left(N_{1} T\right) \exp \left(k \omega_{1}\right) \rightarrow \infty$ as $k \rightarrow \infty$, which is a contradiction.

For case (2), the same arguments can be used.

Now consider case (3). Choose $\eta_{2}>0$ small enough so that

$$
\omega_{2}=\int_{n T}^{(n+1) T}\left(r_{1}-b_{1} l_{1}-\lambda_{1}\left(u^{*}+\eta_{2}\right)\right) d t>0 .
$$


From case (3),

$$
\begin{aligned}
\dot{z}(t) \leq & \left(e_{1} \lambda_{1} x(t)+u e_{2} \lambda_{2} y(t)-m\right) z(t) \\
\leq & \left(-m+e_{1} \lambda_{1} l_{1}+u e_{2} \lambda_{2} l_{2}\right) z(t) \\
= & B z(t) \quad t \neq n T \\
& z\left(t^{+}\right)=z(t)+p \quad t=n T,
\end{aligned}
$$

where $B=-m+e_{1} \lambda_{1} l_{1}+u e_{2} \lambda_{2} l_{2}$. Therefore, $z(t) \leq u(t)$ and $u(t) \rightarrow u^{*}(t)$ as $t \rightarrow \infty$, where $u(t)$ is the solution of the following equation:

$$
\begin{aligned}
\dot{u}(t) & =B u(t) \quad t \neq n T \\
u\left(t^{+}\right) & =u(t)+p \quad t=n T \\
u\left(0^{+}\right) & =z\left(0^{+}\right) \geq 0
\end{aligned}
$$

and $u^{*}(t)=p \exp (B(t-n T)) /(1-\exp (B T))$. Then there exists a $T_{2}>0$, when $t \geq T_{2}$, such that

$$
\begin{gathered}
z(t) \leq u(t)<u^{*}(t)+\eta_{2}, \\
\dot{x}(t) \geq\left(r_{1}-b_{1} l_{1}-\lambda_{1}\left(u^{*}(t)+\eta_{2}\right)\right) x(t) \quad t \neq n T \\
x\left(t^{+}\right)=x(t) \quad t=n T .
\end{gathered}
$$

Let $N_{2} \in N$ and $N_{2} T \geq T_{2}$. Integrating (40) on ( $n T,(n+$ 1)T], $n \geq N_{2}$, the following result can be obtained:

$$
\begin{aligned}
& x((n+1) T) \\
& \quad \geq x(n T) \exp \left(\int_{n T}^{(n+1) T}\left(r_{1}-b_{1} l_{1}-\lambda_{1}\left(u^{*}+\eta_{2}\right)\right) d t\right) \\
& \quad=x(n T) \exp \left(\omega_{2}\right) .
\end{aligned}
$$

Then $x\left(\left(N_{2}+k\right) T\right) \geq x\left(N_{2} T\right) \exp \left(k \omega_{2}\right) \rightarrow \infty$ as $k \rightarrow \infty$, which is a contradiction.

In conclusion, there exist $t_{1}>0$ and $t_{2}>0$ such that $x(t) \geq l_{1}, y(t) \geq l_{2}$.

Second, if $x\left(t_{1}\right) \geq l_{1}$, for all $t>t_{1}$, then the objective has been attained. Otherwise, there exists $t$ such that $x(t)<l_{1}$, for $t>t_{1}$. Let $t^{*}=\inf _{t<t^{*}}\left\{x(t)<l_{1}\right\}$. Then $x\left(t_{1}\right) \geq l_{1}$, for $t \in\left[t_{1}, t^{*}\right)$ and $t^{*} \in\left(n_{1} T,\left(n_{1}+1\right) T\right], n_{1} \in N$, and $x\left(t^{*}\right)=l_{1}$, because $x(t)$ is continuous. Choose $n_{2}, n_{3} \in N$ such that

$$
\begin{gathered}
n_{2} T>T_{2}=\frac{\ln \left(\eta_{1} /(M+p)\right)}{A}, \\
\exp \left(\delta\left(n_{2}+1\right) T\right) \exp \left(n_{3} \omega_{1}\right)>1,
\end{gathered}
$$

where $\delta=r_{1}-b_{1} l_{1}-\lambda_{1} M<0$. Let $T^{\prime}=n_{2} T+n_{3} T$; then there must be a $t^{\prime} \in\left(\left(n_{1}+1\right) T,\left(n_{1}+1\right) T+T^{\prime}\right]$ such that $x\left(t^{\prime}\right) \geq l_{1}$; otherwise $x(t)<l_{1}, t \in\left(\left(n_{1}+1\right) T,\left(n_{1}+1\right) T+T^{\prime}\right]$. Considering (32) with $v\left(\left(n_{1}+1\right) T^{+}\right)=z\left(\left(n_{1}+1\right) T^{+}\right)$,

$$
\begin{aligned}
v(t)= & \left(v\left(\left(n_{1}+1\right) T^{+}\right)-\frac{p}{1-\exp (A T)}\right) \\
& \times \exp \left(A\left(t-\left(n_{1}+1\right) T\right)\right)+v^{*}(t)
\end{aligned}
$$

for $t \in(n T,(n+1) T]$ and $n_{1}+1<n<n_{1}+1+n_{2}+n_{3}$. Then, for $\left(n_{1}+1+n_{2}\right) T \leq t \leq\left(n_{1}+1\right) T+T^{\prime}$,

$$
\begin{gathered}
\left|v(t)-v^{*}(t)\right|<(M+p) \exp \left(A\left(t-\left(n_{1}+1\right) T\right)\right)<\eta_{1} \\
z(t) \leq v(t)<v^{*}(t)+\eta_{1} .
\end{gathered}
$$

It can be concluded that (34) holds for $\left(n_{1}+1+n_{2}\right) T \leq t \leq$ $\left(n_{1}+1\right) T+T^{\prime}$. As in the first step above, it is possible to obtain $x\left(\left(n_{1}+1+n_{2}+n_{3}\right) T\right) \geq x\left(\left(n_{1}+1+n_{2}\right) T\right) \exp \left(n_{3} \omega_{1}\right)$. There are two possible cases for $t \in\left(t^{*},\left(n_{1}+1\right) T\right]$.

Case (a). If $x(t)<l_{1}$ for $t \in\left(t^{*},\left(n_{1}+1\right) T\right]$, then $x(t)<l_{1}$ for $t \in\left(t^{*},\left(n_{1}+1+n_{2}\right) T\right]$. From System (2),

$$
\begin{aligned}
\dot{x}(t) & \geq\left(r_{1}-b_{1} x(t)-\lambda_{1} z(t)\right) x(t) \\
& \geq\left(r_{1}-b_{1} l_{1}-\lambda_{1} M\right) x(t)=\delta x(t) .
\end{aligned}
$$

Integrating (45) on $\left(t^{*},\left(n_{1}+1+n_{2}\right) T\right], x\left(\left(n_{1}+1+n_{2}\right) T\right) \geq$ $l_{1} \exp \left(\delta\left(1+n_{2}\right) T\right)$.

Thus

$$
\begin{aligned}
& x\left(\left(n_{1}+1+n_{2}+n_{3}\right) T\right) \\
& \quad \geq l_{1} \exp \left(\delta\left(1+n_{2}\right) T\right) \exp \left(n_{3} \omega_{1}\right)>l_{1}
\end{aligned}
$$

which is a contradiction.

Let $\bar{t}=\inf _{t>t^{*}}\left\{x(t) \geq l_{1}\right\}$, so that $x(\bar{t})=l_{1}$ and (45) holds on $\left[t^{*}, \bar{t}\right)$. Integrating $(45)$ on $\left[t^{*}, \bar{t}\right)$,

$$
\begin{aligned}
x(t) & \geq x\left(t^{*}\right) \exp \left(\delta\left(t-t^{*}\right)\right) \\
& \geq l_{1} \exp \left(\delta\left(1+n_{2}+n_{3}\right) T\right) \stackrel{\Delta}{\longrightarrow} \bar{l}_{1} .
\end{aligned}
$$

For $t>\bar{t}$, the same arguments can be used because $x(\bar{t}) \geq l_{1}$.

Case (b). There exists a $t^{\prime \prime} \in\left(t^{*},\left(n_{1}+1\right) T\right]$ such that $x\left(t^{\prime \prime}\right) \geq$ $l_{1}$; let $\bar{t}=\inf _{t>t^{*}}\left\{x(t) \geq l_{1}\right\}$; then $x(t)<l_{1}$ for $\left[t^{*}, \bar{t}\right)$ and $x(\bar{t})=l_{1}$. For $t \in\left[t^{*}, \bar{t}\right),(45)$ holds true. Integrating (45) on $\left[t^{*}, \bar{t}\right)$,

$$
x(t) \geq x\left(t^{*}\right) \exp \left(\delta\left(t-t^{*}\right)\right) \geq l_{1} \exp (\delta T)>\bar{l}_{1} .
$$

For $t>\bar{t}$, the same arguments can be used because $x(\bar{t}) \geq l_{1}$.

In summary, $x(\bar{t}) \geq \bar{l}_{1}$ can be obtained for all $t>t_{1}$. In the same way, it can be proved that $y(\bar{t}) \geq \bar{l}_{2}$ for all $t>t_{2}$. This completes the proof.

\section{Numerical Analysis}

3.1. The Impulsive Effect and Optimal Foraging. To study the population dynamics of a three-species ecological model with impulsive effect, the solution of System (2) with initial conditions in the first quadrant is obtained numerically for a biologically feasible range of parametric values, and the bifurcation diagram provides a summary of the basic population dynamic behavior of the system. 


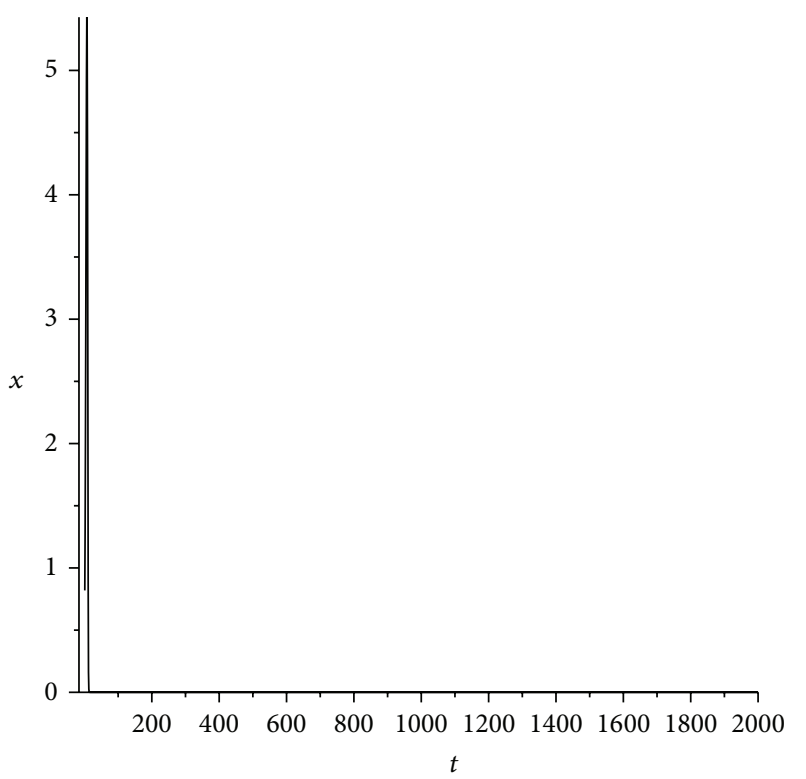

(a)

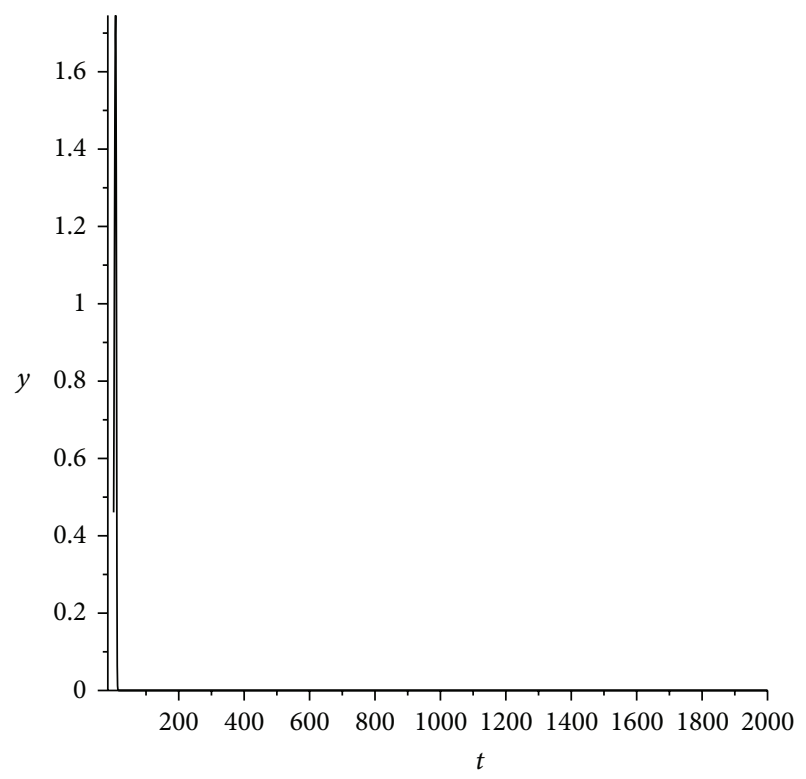

(b)

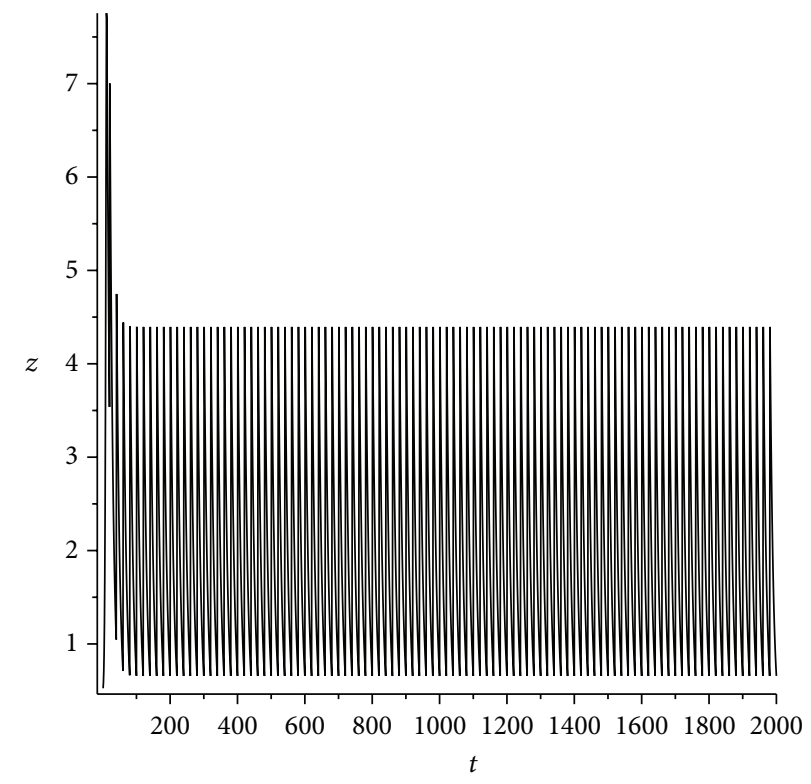

(c)

Figure 1: Time series of System (2) when $u=0.8$ and $p=4.2$.

Now two different control parameters will be discussed, the number of predators released, $p$, and the probability $u$. Other parameters are set to

$$
\begin{array}{rlll}
a_{1}=0.35, & a_{2}=0.4, & r_{1}=0.9, & r_{2}=0.8, \\
k_{0}=15, & k_{1}=20, & k_{2}=12, & k_{3}=15, \\
b_{1}=0.045, & b_{2}=0.2, & h_{1}=0.8, & h_{2}=0.45, \\
e_{1}=0.5, & e_{2}=0.6, & m=0.1, & T=20 .
\end{array}
$$

From Theorem 7, it is known that the prey-eradication periodic solution $\left(0,0, z^{*}(t)\right)$ is locally asymptotically stable provided that $p>p_{\max } \approx 4.098648$. Figure 1 shows a typical prey-eradication periodic solution of System (2), in which it can be observed that the variable $z$ oscillates in a stable cycle. At the same time, the prey types $x$ and $y$ rapidly diminish and go to zero beyond $p_{\max } \approx 4.098648$. If the number of predators released, $p$, is less than $p_{\max }$, the prey-eradication solution becomes unstable. It is, however, possible that the two prey types and the predator can coexist in a stable positive periodic solution. In other words, the system can be permanent when the number of predators released, $p$, is less than $p_{\max }$. 


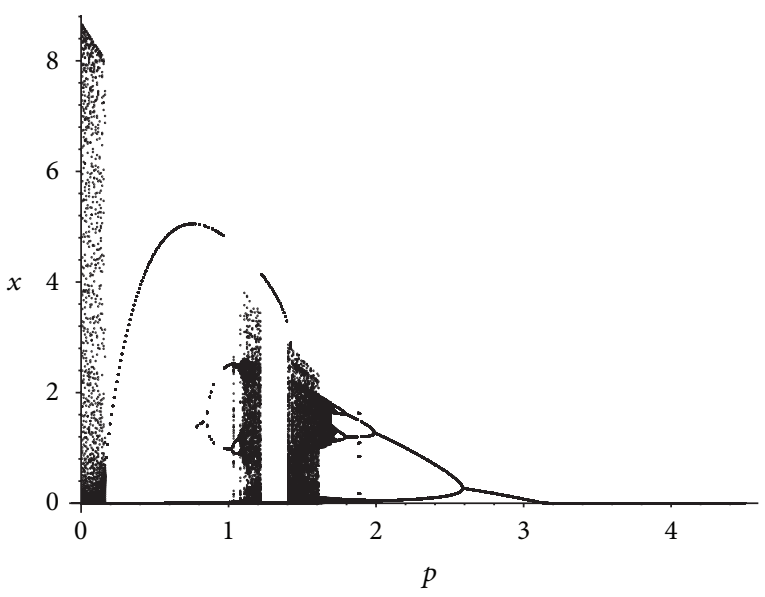

(a)

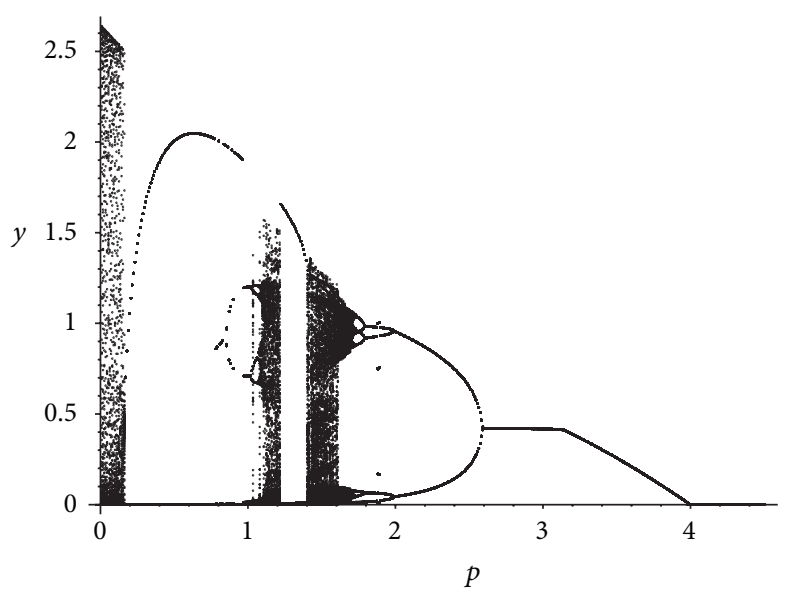

(b)

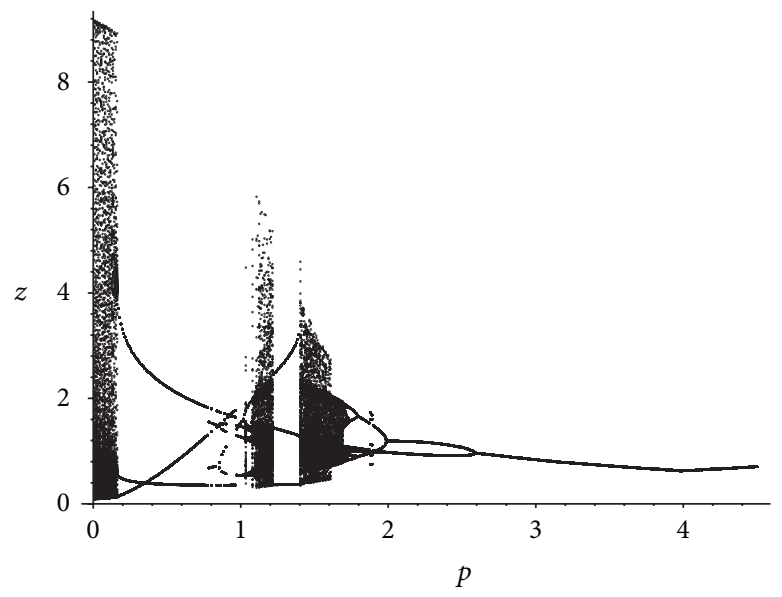

(c)

FIgURE 2: Bifurcation diagram of System (2) with initial conditions $x(0)=0.3, y(0)=0.5, z(0)=0.3, u=0.8$, and $0.009 \leq p \leq 4.5$.

Next, the bifurcation diagrams for the control parameter $p$ will be examined. Figure 2 is plotted as a function of the bifurcation parameter $p$ and shows that the system has rich population dynamic behavior consistent with the theoretical analysis, such as period-halving bifurcation (see Figure 3), a chaotic band, a periodic window, and chaotic crises. Furthermore, Theorem 8 indicates that the system is permanent when the value of $p$ is less than some critical value. When the value of $p$ is in the interval $[0.009,3.257895]$, the two prey types and one predator can coexist. When the value of $p$ is in the interval $[3.257895,4.098648]$, the prey $x$ will become extinct rapidly, but the prey $y$ and the predator $z$ can coexist. These results may show that prey $x$ is inferior to prey $y$ in its ability to reproduce or prey $x$ is a favorite food of predator $z$. When the number of predators released is greater than some critical value, all species in the system will become extinct. All these results demonstrate the effectiveness of mathematical analysis for understanding such systems.

The next question is how $u$ impacts the complex population dynamics. In Figure 4, when prey and predator populations are plotted as a function of the probability $u$, the value of $p$ is 1.45 . In the former case, it is assumed that the foraging behavior of predator $z$ follows optimal foraging theory [16-18] and prey $x$ is more beneficial for predator $z$ than prey $y$. In other words, the more beneficial prey $x$ is always included in the predator's diet, but if the density of prey $x$ falls below a critical threshold or goes to zero, prey $y$ is included with probability one. From Figure 4, it can be clearly observed that the two prey types and the predator can coexist in the intervals $[0,0.1136]$ and $[0.7215,0.8792]$, where the system dynamics can be chaotic, periodic, or nonperiodic. In the interval $(0.1136,0.7215)$, prey $x$ goes extinct, while prey $y$ and predator $z$ can coexist stably. This means that if the more beneficial prey $x$ disappears, prey $y$ alone can support the population of predator $z$. As $u$ increases, prey $y$ goes extinct, but prey $x$ and predator $z$ can coexist stably.

3.2. The Largest Lyapunov Exponent. Deterministic chaos is an important problem that is solved by measuring the largest 


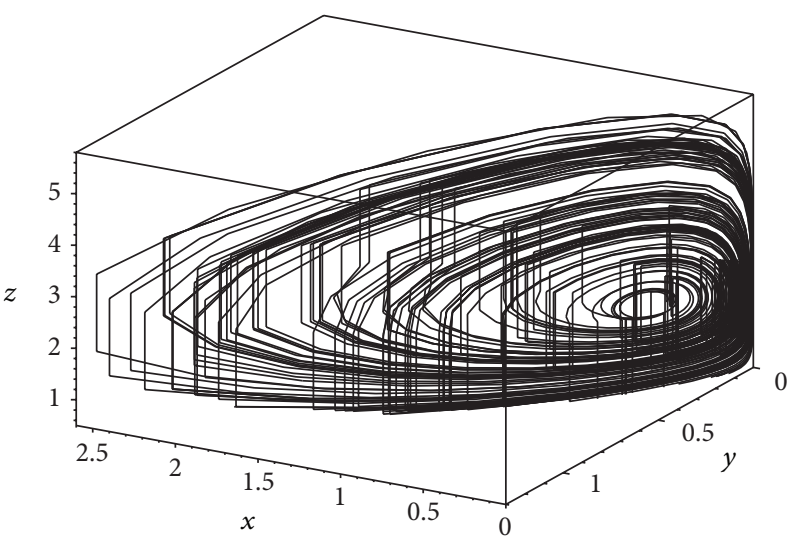

(a)

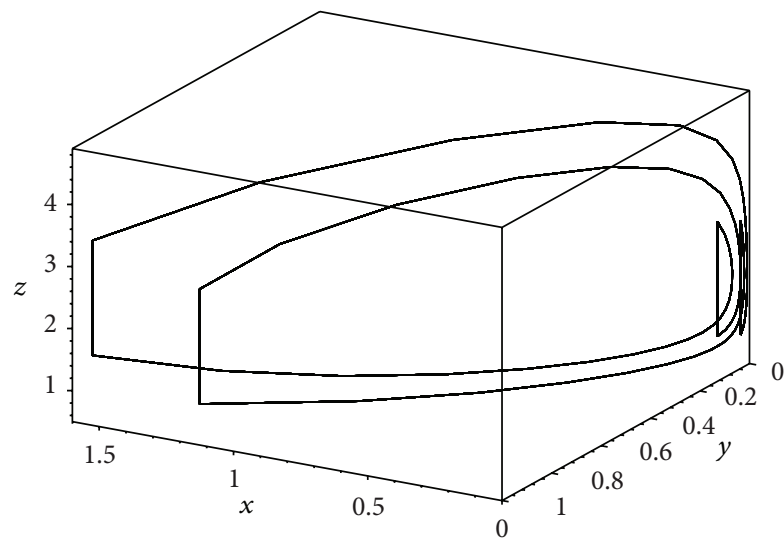

(c)

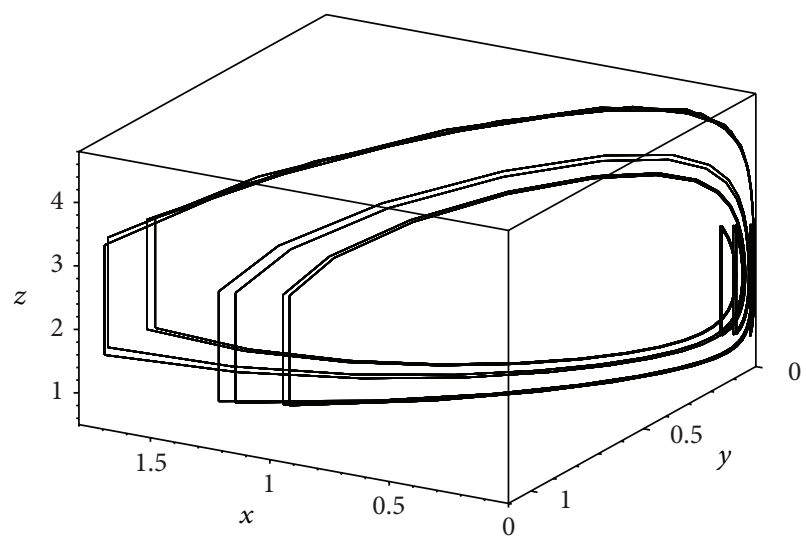

(b)

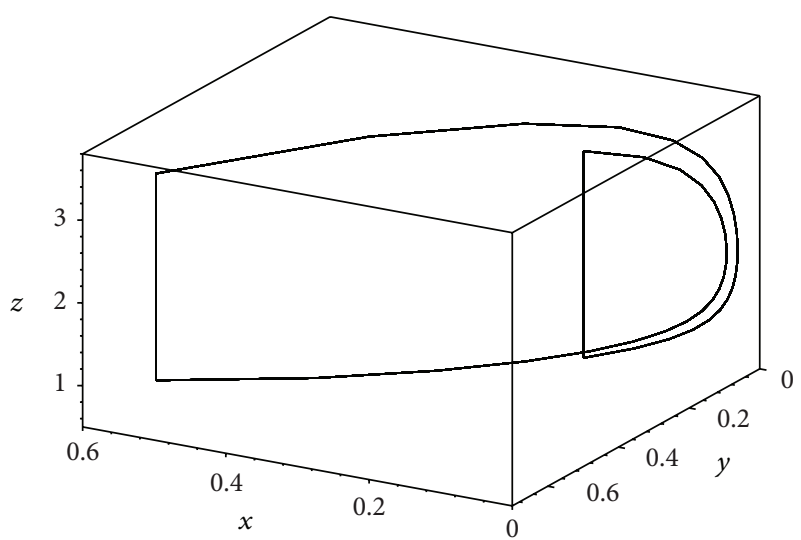

(d)

FIgURE 3: Transition from chaos to period-halving in System (2): (a) chaos when $p=1.5$; (b) 8T-periodic solution when $p=1.73$; (c) 4T-periodic solution when $p=1.85$; (d) 2T-periodic solution when $p=2.5$.

Lyapunov exponent [31-36]. Based on research by various investigators, these results have confirmed the importance of detecting and exploring chaos. In this paper, the largest Lyapunov exponents for chaotic system (2) are examined. The largest Lyapunov exponents take into account the average exponential rates of divergence or convergence of nearby orbits in phase space $[31,32]$. If the attractor is chaotic, the largest Lyapunov exponent must be positive, which implies a stable or a periodic state. In Figure 2, the corresponding largest Lyapunov exponent $([0 \leq p \leq 3])$ can be calculated for System (2) (see Figure 5).

3.3. The Strange Attractor and Power Spectra. To study the properties of strange attractors, commonly used methods such as power spectra can be used $[35,36]$. A power spectrum was calculated using 4096 points corresponding to the time series of the variable $x$ with time increment $\Delta t=0.5[35,36]$. For strange attractors (a) and (b), it is known that the value of the largest Lyapunov exponent for the strange attractor (a) is 0.25603 , while for (b) the computed largest Lyapunov exponent is 0.30567 . Therefore, strange attractors (a) and (b) are chaotic attractors. Moreover, the strange attractor (b) displays more chaotic dynamics than (a) because its positive exponent is larger than that of (a). In addition, the spectra of strange attractors (a) and (b) consist of strong broadband components and sharp peaks (Figures 6(c) and 6(d)) These results conform to the observation that strange attractors (a) and (b) arise from some weak limit cycles which can lose stability due to noise.

\section{Conclusions and Remarks}

Complex population dynamics of a three-species ecological model with optimal foraging and impulsive control strategy have been investigated both numerically and analytically. The periodic solution has been shown to be globally asymptotically stable by use of the Floquet theorem and small-amplitude perturbations, if $p>\max \left(r_{1} k_{0} T m / \lambda_{1} k_{1}, r_{2} k_{2} T m / u \lambda_{2} k_{3}\right)$ and $p \exp (-m T) /$ $(1-\exp (-m T))>\max \left(r_{1}\left(1+h_{1} \lambda_{1} M+u h_{2} \lambda_{2} M\right) / \lambda_{1}, r_{2}(1+\right.$ 


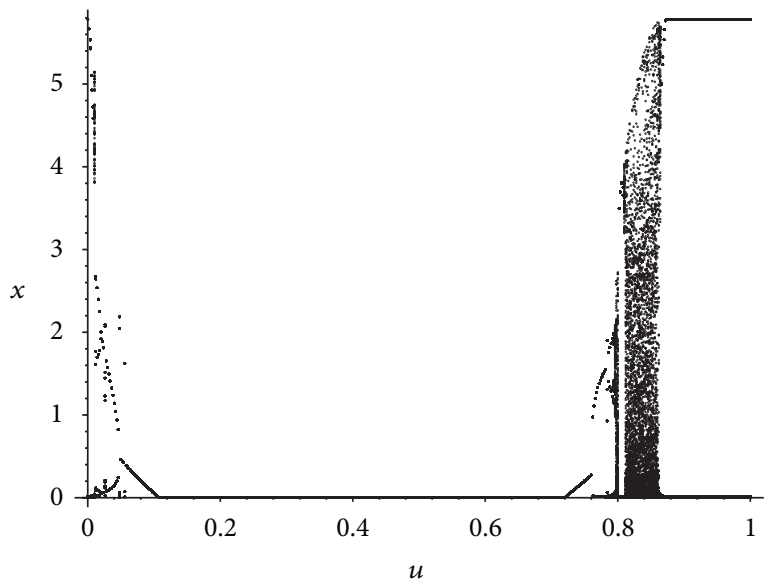

(a)

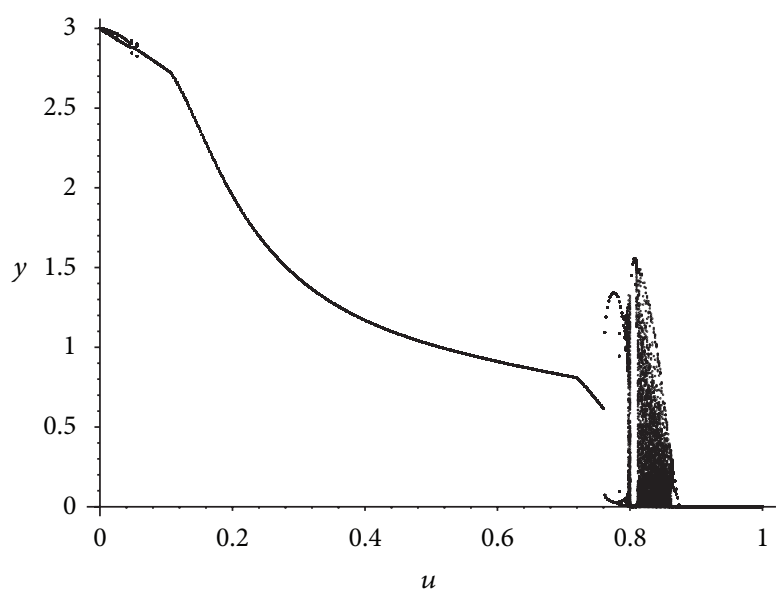

(b)

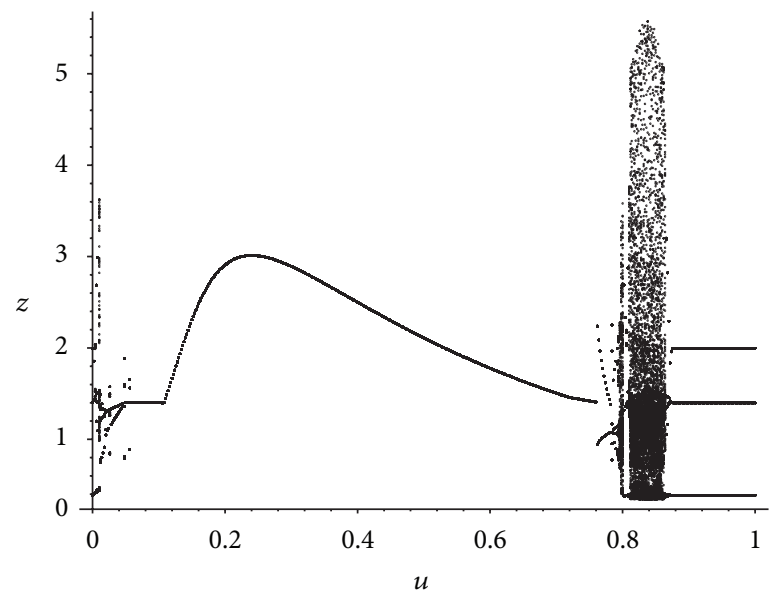

(c)

FIGURE 4: Bifurcation diagram of System (2) with initial conditions $p=1.45, x(0)=0.3, y(0)=0.5, z(0)=0.3$, and $0 \leq u \leq 1$.

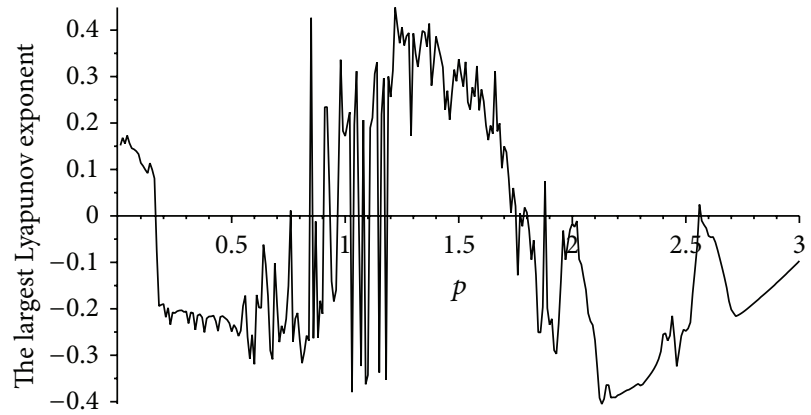

FiguRE 5: The largest Lyapunov exponents for System (2) with $p$ varying from 0 to 3.

$\left.\left.h_{1} \lambda_{1} M+u h_{2} \lambda_{2} M\right) / u \lambda_{2}\right)$. At the same time, using the method of comparison involving multiple Lyapunov functions, the permanence of the system can be proved. Bifurcation diagrams of the impulsive perturbation $p$ and the probability parameter $u$ have also been obtained. The bifurcation diagrams of $p$ have shown that dynamic complexity exists in System (2), including chaotic behavior, periodic windows, chaotic bands, chaotic crises, and period-halving bifurcations. The bifurcation diagrams of $u$ indicate that optimal foraging promotes species coexistence and that if the more beneficial prey goes extinct, the less beneficial prey can support the predator so that it will not die out. In addition, the presence of chaotic dynamics was confirmed, and the qualitative nature of strange attractors was investigated using 


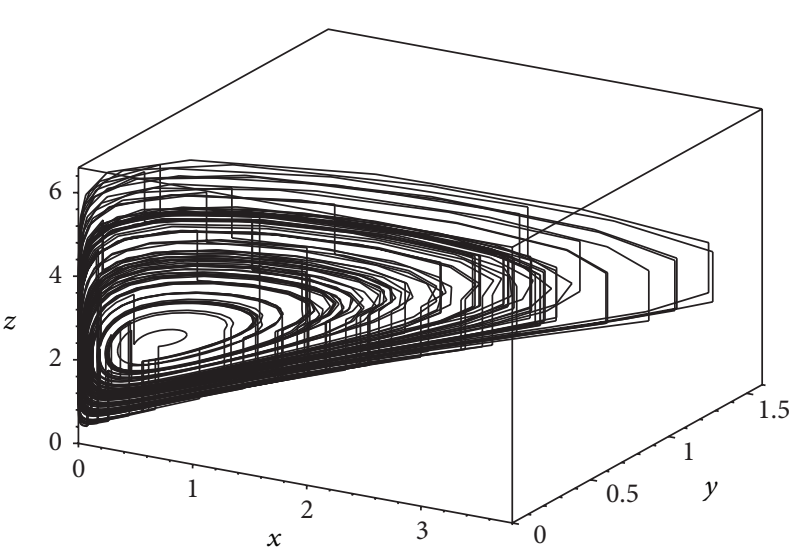

(a)

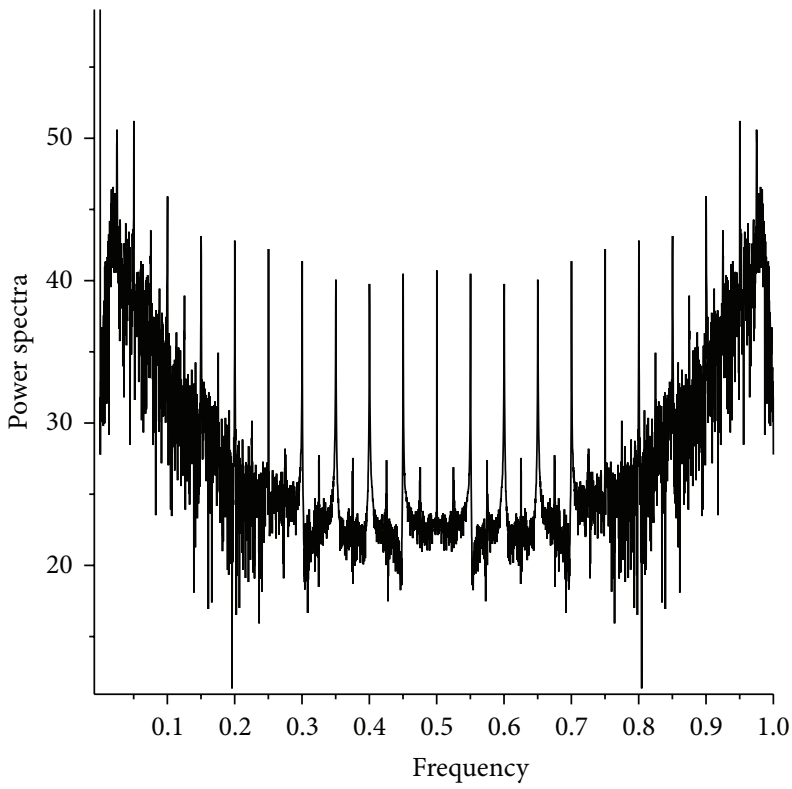

(c)

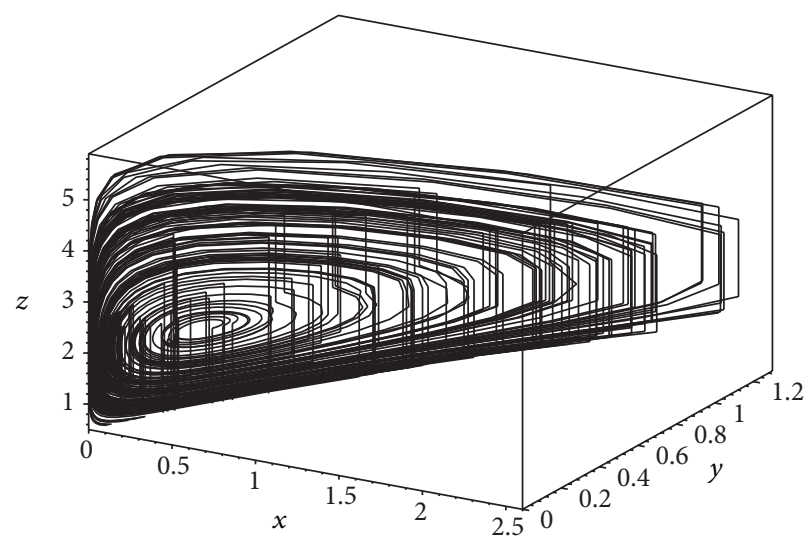

(b)

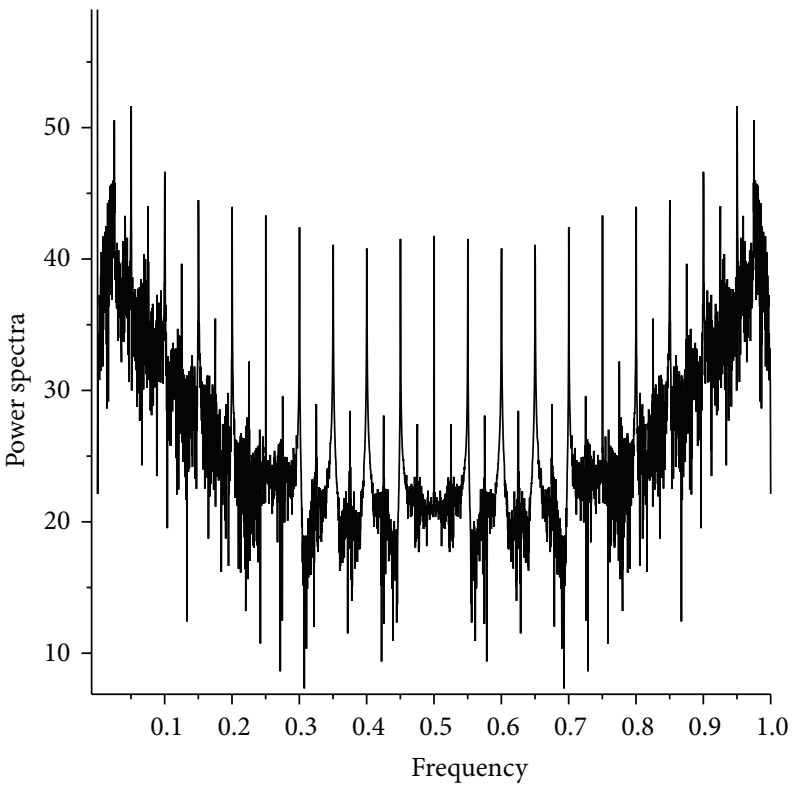

(d)

FIGURE 6: Strange attractors and power spectra: (a)strange attractor when $p=1.2$, (b) strange attractor when $p=1.51$, (c) power spectrum of attractor (a), and (d) power spectrum of attractor (b).

computer simulations of the largest Lyapunov exponents and Fourier spectra. All these results may be useful in the study of the dynamic complexity of ecosystems.

\section{Conflict of Interests}

The authors declare that there is no conflict of interests regarding the publication of this paper.

\section{Acknowledgments}

This work was supported by the National Natural Science Foundation of China (Grant nos. 31370381 and 31170338), by the Key Program of Zhejiang Provincial Natural Science Foundation of China (Grant no. LZ12C03001), and by the
National Key Basic Research Program of China (973 Program, Grant no. 2012CB426510).

\section{References}

[1] R. M. May, "Will a large complex system be stable?" Nature, vol. 238, no. 5364, pp. 413-414, 1972.

[2] R. M. May, "Simple mathematical models with very complicated dynamics," Nature, vol. 261, no. 5560, pp. 459-467, 1976.

[3] R. M. May, "Biological populations with nonoverlapping generations: stable points, stable cycles, and chaos," Science, vol. 186, no. 4164, pp. 645-647, 1974.

[4] H. Zhu, S. A. Campbell, and G. S. K. Wolkowicz, "Bifurcation analysis of a predator-prey system with nonmonotonic functional response," SIAM Journal on Applied Mathematics, vol. 63, no. 2, pp. 636-682, 2002. 
[5] S.-B. Hsu, T.-W. Hwang, and Y. Kuang, "Global analysis of the Michaelis-Menten-type ratio-dependent predator-prey system," Journal of Mathematical Biology, vol. 42, no. 6, pp. 489506, 2001.

[6] Y. Do, H. Baek, Y. Lim, and D. Lim, "A three-species food chain system with two types of functional responses," Abstract and Applied Analysis, vol. 2011, Article ID 934569, 16 pages, 2011.

[7] S. Lv and M. Zhao, "The dynamic complexity of a three species food chain model," Chaos, Solitons and Fractals, vol. 37, no. 5, pp. 1469-1480, 2008.

[8] W. Wang, J. Shen, and J. J. Nieto, "Permanence and periodic solution of predator-prey system with Holling type functional response and impulses," Discrete Dynamics in Nature and Society, vol. 2007, Article ID 81756, 15 pages, 2007.

[9] L. Zhang and M. Zhao, "Dynamic complexities in a hyperparasitic system with prolonged diapause for host," Chaos, Solitons and Fractals, vol. 42, no. 2, pp. 1136-1142, 2009.

[10] R. K. Upadhyay and R. K. Naji, "Dynamics of a three species food chain model with Crowley-Martin type functional response," Chaos, Solitons and Fractals, vol. 42, no. 3, pp. 13371346, 2009.

[11] M. Zhao, L. Zhang, and J. Zhu, "Dynamics of a host-parasitoid model with prolonged diapause for parasitoid," Communications in Nonlinear Science and Numerical Simulation, vol. 16, no. 1, pp. 455-462, 2011.

[12] C. Wei and L. Chen, "A delayed epidemic model with pulse vaccination," Discrete Dynamics in Nature and Society, vol. 2008, Article ID 746951, 12 pages, 2008.

[13] J. Jiao, S. Cai, and L. Chen, "Analysis of a stage-structured predatory-prey system with birth pulse and impulsive harvesting at different moments," Nonlinear Analysis: Real World Applications, vol. 12, no. 4, pp. 2232-2244, 2011.

[14] R. Shi and L. Chen, "Stage-structured impulsive SI model for pest management," Discrete Dynamics in Nature and Society, vol. 2007, Article ID 97608, 11 pages, 2007.

[15] H.-F. Huo, Z.-P. Ma, and C.-Y. Liu, "Persistence and stability for a generalized Leslie-Gower model with stage structure and dispersal," Abstract and Applied Analysis, vol. 2009, Article ID 135843, 17 pages, 2009.

[16] H. Yu, S. Zhong, and R. P. Agarwal, "Mathematics analysis and chaos in an ecological model with an impulsive control strategy," Communications in Nonlinear Science and Numerical Simulation, vol. 16, no. 2, pp. 776-786, 2011.

[17] L. Wang, L. Chen, and J. J. Nieto, "The dynamics of an epidemic model for pest control with impulsive effect," Nonlinear Analysis: Real World Applications, vol. 11, no. 3, pp. 1374-1386, 2010.

[18] H. Yu, S. Zhong, R. P. Agarwal, and S. K. Sen, "Effect of seasonality on the dynamical behavior of an ecological system with impulsive control strategy," Journal of the Franklin Institute: Engineering and Applied Mathematics, vol. 348, no. 4, pp. 652670, 2011.

[19] J. Luo, "Permanence and extinction of a generalized Gause-type predator-prey system with periodic coefficients," Abstract and Applied Analysis, vol. 2010, Article ID 845606, 24 pages, 2010.

[20] X. Wang, W. Wang, and X. Lin, "Dynamics of a two-prey one-predator system with Watt-type functional response and impulsive control strategy," Chaos, Solitons and Fractals, vol. 40, no. 5, pp. 2392-2404, 2009.

[21] R. D. Holt, "Predation, apparent competition and the structure of prey communities," Theoretical Population Biology, vol. 12, no. 2, pp. 197-229, 1977.
[22] H. Werner, "Optimal foraging and the size selection of prey by the bluegill sunfish (Lepomis macrochirus)," Ecology, vol. 55, pp. 1042-1052, 1974.

[23] E. L. Charnov, "Optimal foraging: attack strategy of a mantid," The American Naturalist, vol. 110, no. 971, pp. 141-151, 1976.

[24] D. W. Stephens and J. R. Krebs, Foraging Theory, Princeton University Press, Princeton, NJ, USA, 1986.

[25] V. Křivan and J. Eisner, "Optimal foraging and predator-prey dynamics III," Theoretical Population Biology, vol. 63, no. 4, pp. 269-279, 2003.

[26] V. Křivan, "Optimal foraging and predator-prey dynamics," Theoretical Population Biology, vol. 49, no. 3, pp. 265-290, 1996.

[27] V. Krrivan and A. Sikder, "Optimal foraging and predator-prey dynamics, II," Theoretical Population Biology, vol. 55, no. 2, pp. 111-126, 1999.

[28] V. Lakshmikantham, D. D. Baĭnov, and P. S. Simeonov, Theory of Impulsive Differential Equations, vol. 6, World Scientific, Singapore, 1989.

[29] D. D. Baĭnov and P. S. Simeonov, Impulsive Differential Equations: Asymptotic Properties of the Solutions, World Scientific, Singapore, 1993.

[30] D. D. Bainov and V. C. Covachev, Impulsive Differential Equations with a Small Parameter, vol. 24, World Scientific, Singapore, 1994.

[31] S. Lv and M. Zhao, "The dynamic complexity of a hostparasitoid model with a lower bound for the host," Chaos, Solitons and Fractals, vol. 36, no. 4, pp. 911-919, 2008.

[32] M. Zhao and S. Lv, "Chaos in a three-species food chain model with a Beddington-DeAngelis functional response," Chaos, Solitons and Fractals, vol. 40, no. 5, pp. 2305-2316, 2009.

[33] M. Zhao and L. Zhang, "Permanence and chaos in a hostparasitoid model with prolonged diapause for the host," Communications in Nonlinear Science and Numerical Simulation, vol. 14, no. 12, pp. 4197-4203, 2009.

[34] L. Zhu and M. Zhao, "Dynamic complexity of a host-parasitoid ecological model with the Hassell growth function for the host," Chaos, Solitons and Fractals, vol. 39, no. 3, pp. 1259-1269, 2009.

[35] H. Yu, S. Zhong, R. P. Agarwal, and S. K. Sen, "Threespecies food web model with impulsive control strategy and chaos," Communications in Nonlinear Science and Numerical Simulation, vol. 16, no. 2, pp. 1002-1013, 2011.

[36] H. Yu, S. Zhong, and M. Ye, "Dynamic analysis of an ecological model with impulsive control strategy and distributed time delay," Mathematics and Computers in Simulation, vol. 80, no. 3, pp. 619-632, 2009. 


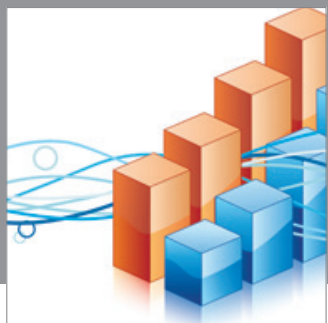

Advances in

Operations Research

mansans

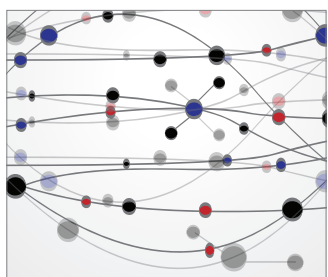

The Scientific World Journal
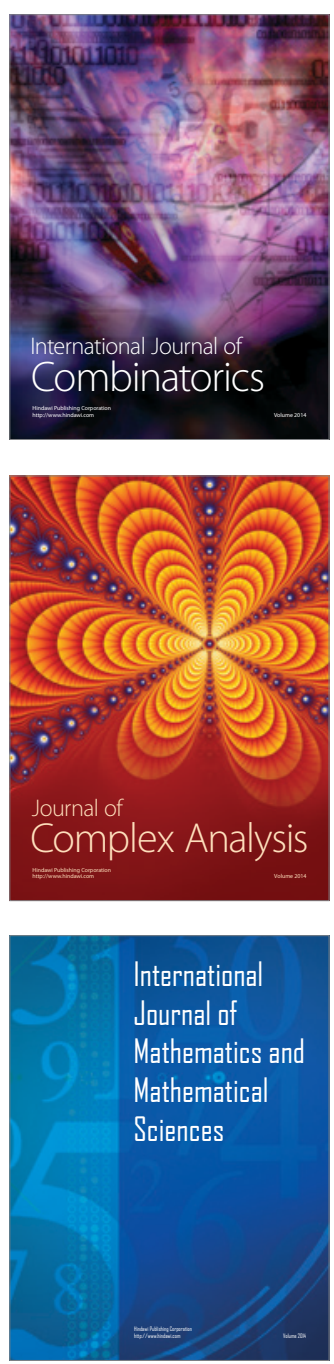
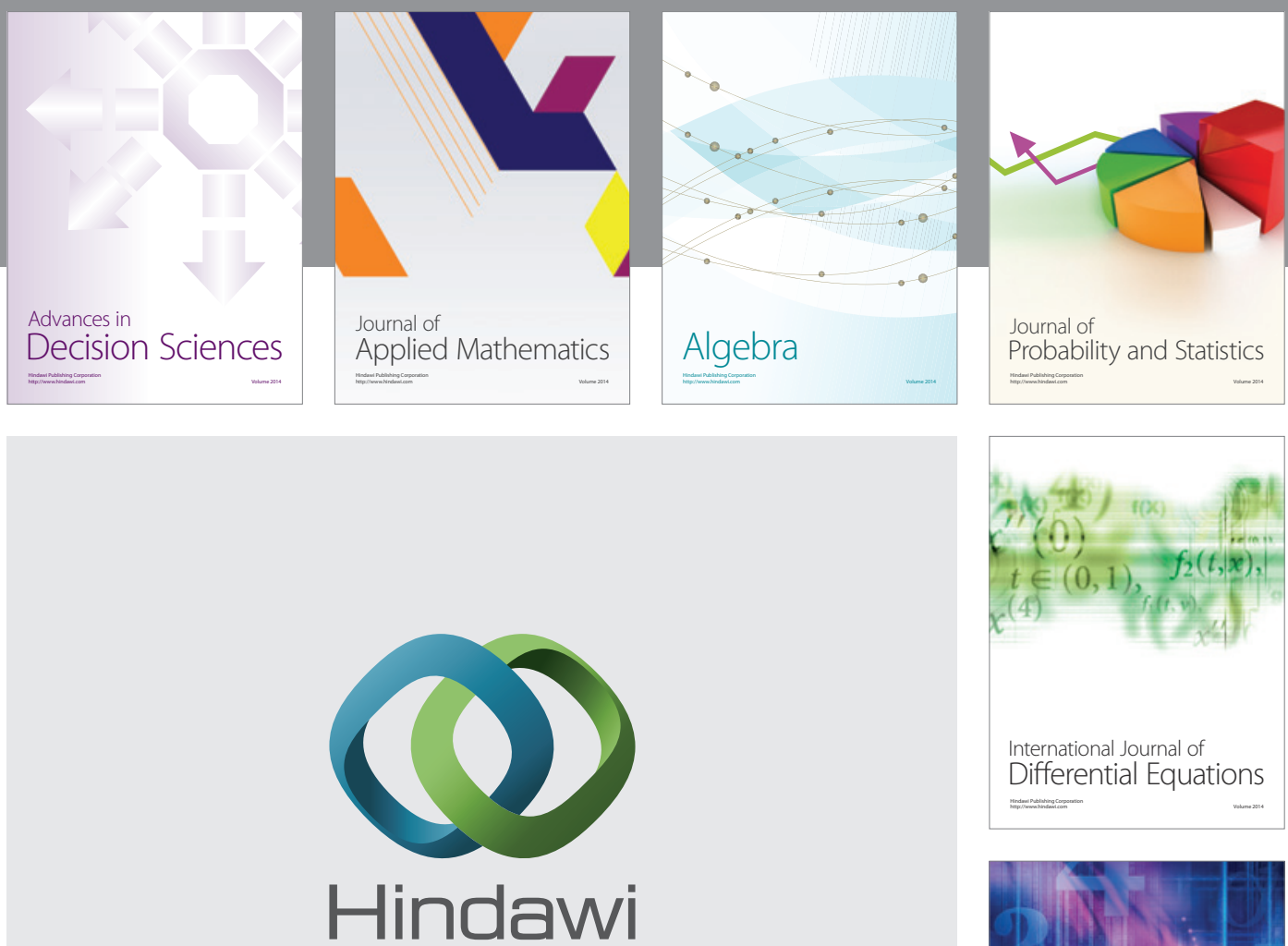

Submit your manuscripts at http://www.hindawi.com
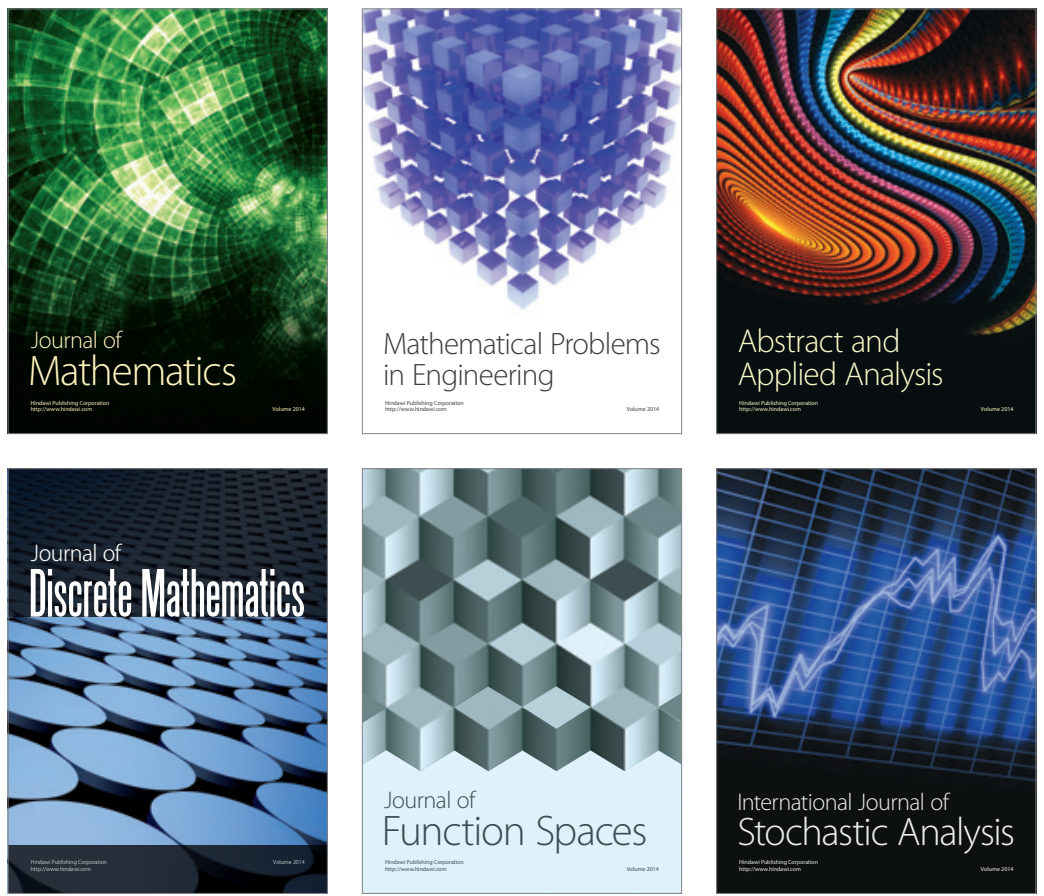

Journal of

Function Spaces

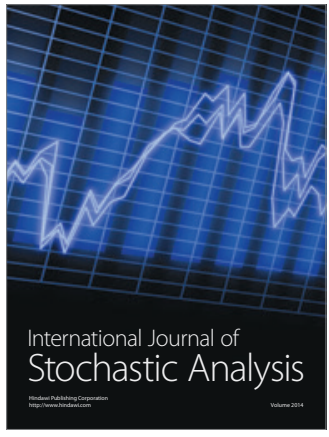

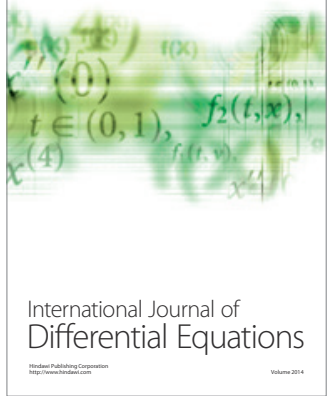
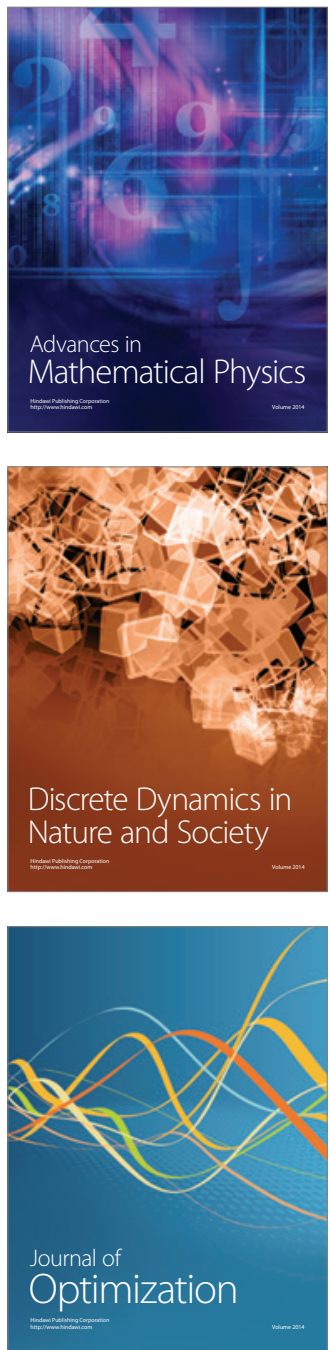